\section{INSIDE LI}

doi:10.1038/labinvest.2012.120
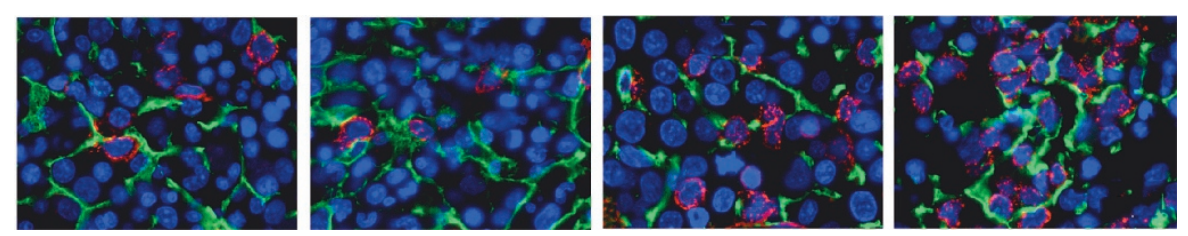

\section{Microenvironment architecture in normal and myelodysplastic bone marrow}

See page 1330

The bone marrow microenvironment provides a crucial niche for nurturing the development of bone marrow cells from $\mathrm{CD}_{3} 4^{+}$hematopoietic progenitor/ stem cells (HSPCs). However, subtleties in the relationship between the bone marrow microenvironment and HSPCs are only now being appreciated. The bone marrow microenvironment is complex and consists of mesenchymal stromal cells (MSCs), blood vessels, adipocytes, macrophages, and trabecular bone. Myelodysplastic syndrome (MDS) is a group of clinically and cytogenetically heterogeneous clonal bone marrowfailure disorders with potential to progress to acute myelogenous leukemia (AML). Because MSCs from MDS and AML patients exhibit structural chromosomal abnormalities that differ from those of the hematopoietic clone, these cells probably contribute to the development of MDS and AML.

To investigate the relationship between the bone marrow microenvironment and HSPCs, Flores-Figueroa and colleagues performed a methodical morphologic analysis of various components within bone marrow. Their analysis led to the development of a model for investigating how changes in the bone marrow microenvironment may facilitate the development of MDS. They found that $\mathrm{CD} 271^{+}$MSCs form a network throughout the microenvironment that interacts directly with $\mathrm{CD} 34^{+} \mathrm{HSPCs}$ and facilitates interactions between
HSPCs and the microenvironment. In MDS, the MSCs are expanded and exhibit increased expression of CXCL12, a potential source of abnormal survival proliferation signaling for CD $34^{+} \mathrm{HSPCs}$. Increased contact between HSPCs and CXCL12-expressing MSCs may be the fertile ground that spawns the abnormal localization of immature precursors that is characteristic of MDS. This study illustrates how pathologists can contribute to a better understanding of various preneoplastic and neoplastic processes.

\section{Regulation of vascular calcification by miRNAs}

See page 1250

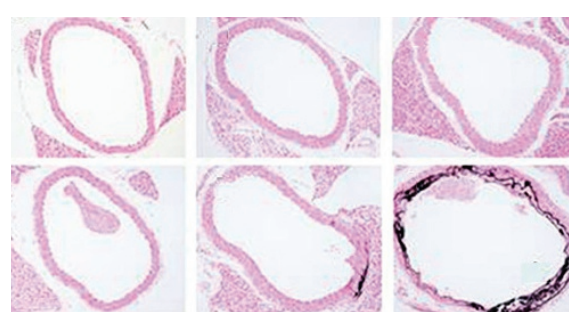

MicroRNAs (miRNAs) are small, endogenous, noncoding RNAs that target coding messenger RNAs (mRNAs) for degradation and have the potential to control gene expression of virtually any gene. Each miRNA has the potential to target multiple mRNAs. The field is still young, but it is becoming clear that miRNAs are involved in controlling virtually all biological processes.

Vascular smooth muscle calcification is commonly associated with vascular disease and is a common complication of chronic kidney disease (CKD). Klotho is a coreceptor of fibroblast growth factor (FGF)-23. FGF-23 plays important roles in control of phosphate (Pi) and calcium
(Ca) concentrations. Klotho homozygous knockout mice $(\mathrm{kl} / \mathrm{kl})$ develop vascular calcification and other CKD-associated bone and mineral disorders and are therefore an excellent model in which to study vascular smooth muscle calcification associated with CKD. Gui et al hypothesized that miRNAs might have a role in regulation of vascular smooth muscle calcification.

The authors used miRNA profiling of vascular tissues from $\mathrm{kl} / \mathrm{kl}$ mice, which develop aortic smooth muscle calcification by 3 weeks, to identify several miRNAs with dysregulated expression in $\mathrm{kl} / \mathrm{kl}$ mice as compared with wild-type mice. Bioinformatics analysis of targets of miRNAs that were overexpressed revealed that four miRNAs targeted $\mathrm{Ca}^{2+}$ efflux channel mRNAs. This led to the hypothesis that decreased expression of $\mathrm{Ca}^{2+}$ efflux channels due to miRNA targeting could lead to increased intracellular $\mathrm{Ca}^{2+}$ and calcification. This was confirmed experimentally by forced expression of miRNAs targeting $\mathrm{Ca}^{2+}$ efflux channel mRNAs in vascular smooth muscle cells, which resulted in increased intracellular $\mathrm{Ca}^{2+}$ levels. Further studies will be required to determine the overall contribution of miRNA targeting of $\mathrm{Ca}^{2+}$ efflux channel mRNAs to vascular calcification.

\section{Evaluation of myofibroblasts in interstitial lung disease}

\section{See page 1270}

Interstitial lung disease refers to a group of lung diseases that affect the interstitium. The group includes idiopathic pulmonary fibrosis, nonspecific interstitial pneumonia, connective-tissue disease-associated interstitial lung diseases, sarcoidosis, asbestosis, and allergic alveolitis. Interstitial lung disease is characterized by various degrees of fibrosis, consisting at the cellular level of proliferation of fibroblasts and myofibroblasts. 


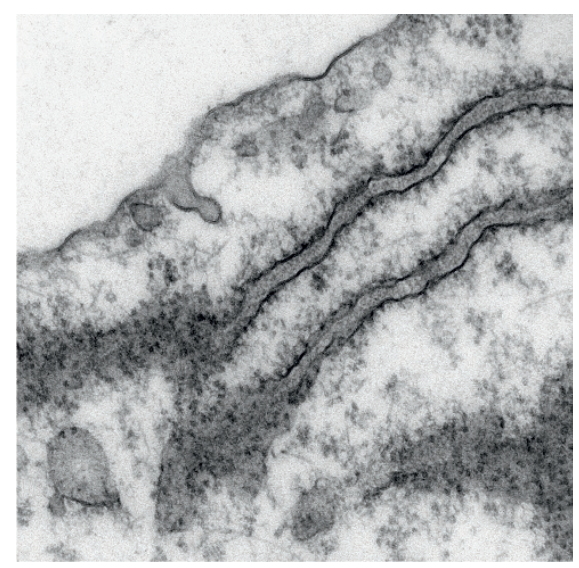

To obtain a better understanding of the role of myofibroblasts in interstitial lung diseases, Karvonen et al developed a technique to isolate myofibroblasts from routine, small-volume, bronchoalveolar-lavage specimens. They hypothesized that myofibroblasts would differ between different types of interstitial lung diseases. They analyzed myofibroblasts from a wide variety of interstitial lung diseases and found that they had varying numbers and types of fibronexus structures, which contain actin and fibronectin and are among the defining electron microscopic features of myofibroblasts. Immunoelectron microscopic analysis enabled the authors to quantitate the amount of a-smooth muscle actin (a-SMA) and fibronectin, which varied within and between different interstitial lung diseases. Overall, interstitial lung disease specimens contained more a-SMA than normal lung specimens. Perhaps the most striking finding was in an invasion assay in which the authors found that myofibroblasts from interstitial pulmonary fibrosis had the greatest invasive capacity and that invasion correlated with the level of actin. Overall, these results suggest that other functional and morphological studies of myofibroblasts derived from bronchoalveolar-lavage specimens of patients with lung diseases may shed light on the pathogenesis and identify therapeutic opportunities.

\section{nature.com/pathology}

Autocrine MET activation drives acute myelogenous leukemia To gain insight into proteins that drive growth and survival in acute myelogenous leukemia (AML), Kentsis et al, as reported in a recent letter in Nature Medicine, used a loss-of-function RNA interference genomic screen in an AML cell line. Hepatocyte growth factor (HGF) was among the genes most substantially required for proliferation and survival. The authors demonstrated that HGF activated mesenchymal-epithelial transition (MET),

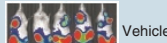

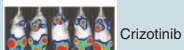

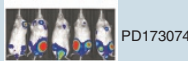
1111 combination the HGF growth factor receptor through an autocrine mechanism. However, pharmacological inhibition with crizotinib, a small-molecule tyrosine kinase inhibitor that targets MET, was short lived, with acquired resistance developing within 6 days in vitro due to compensatory HGF overexpression. This was dependent on fibroblast growth factor receptor 1 (FGFR1) activation in stem cell leukemia with an FGFR1 translocation. Targeting FGFR1 and HGF eliminated resistance synergistically. Because autocrine activation of growth factor receptors is a common pathogenetic mechanism in cancers, this study provides crucial information about how to target compensatory autocrine activation of crucial cancer signaling pathways. Nature Medicine 2012;18: 1118-1122; doi:10.1038/nm.2819

\section{Pharmacological targeting of radiation toxicity Radiation} toxicity is a major problem associated with total body irradiation (TBI). To identify genes involved in radioprotection, Geiger et al devised an ingenious retroviral insertional mutagenesis scheme in mice, described in a recent letter in Nature Medicine. They identified a mutant that resulted in increased expression of

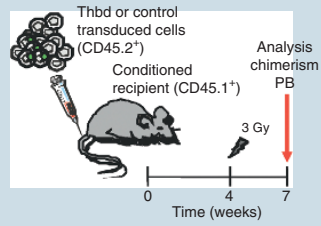
thrombomodulin (Thbd), resulting in significant radioprotection of bone marrow but not the gastrointestinal tract. Increased expression of Thbd resulted in activation of the Thbd-activated protein C (aPC) pathway, although determining precisely how this pathway protects bone marrow from TBI will require further study. Infusion of aPC was found to be radioprotective of bone marrow, suggesting an immediate therapeutic application for transplant patients who receive $\mathrm{TB}$.

Nature Medicine 2012;18:1123-1129; doi:10.1038/nm.2813

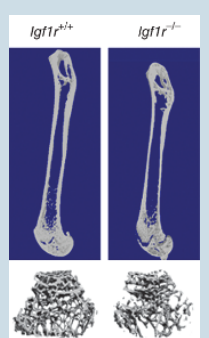

IGF-1 maintains bone mass Insulin-like growth factor 1 (IGF-1), the most abundant growth factor in the bone matrix, has been implicated in bone formation in vitro. On the basis of these observations, Xian et al, as reported in a recent article in Nature Medicine, investigated the role of IGF-1 in maintenance of bone mass in vivo. They determined that IGF-1 was released by osteoclasts during bone resorption and regulated differentiation of mesenchymal stem cells into osteoblasts, thus coupling bone resorption to bone formation. IGF-1 levels decrease with age and are lower in osteoporotic patients with hip fractures, linking IGF-1 levels to bone loss. IGF-1 deposition in the bone matrix is regulated by IGF binding protein 3 (IGFBP3). Infusion of IGF-1 plus IGFBP3 enhances trabecular bone formation, suggesting a therapeutic approach to delaying or preventing osteoporosis. Nature Medicine 2012;18:1095-1101; doi:10.1038/nm.2793

\section{New biomarker of chemotherapy resistance in}

breast cancer In a recent article in Nature Medicine, Balko et al explain how they used gene expression profiling to identify decreased expression of dual-specificity protein

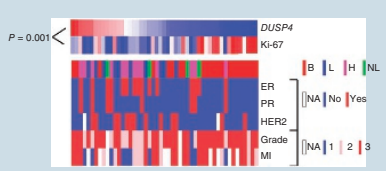
phosphatase 4 (DUSP4) as a mechanism of resistance to neoadjuvant chemotherapy (NAC). They demonstrated that decreased expression of DUSP4, a negative regulator of the Ras-ERK pathway, correlated with Ras-ERK pathway activation in residual basal-like breast cancers (BLBCS). This suggested that loss of DUSP4 contributed to Ras-ERK pathway activation and NAC resistance. They confirmed this by demonstrating that mitogen-activated protein kinase kinase inhibitors, which inhibit the Ras-ERK pathway, synergized with docetaxel in BLBC xenografts, suggesting that this treatment strategy might work in NAC in humans.

NatureMedicine 2012;18:1052-1059; doi:10.1038/nm.2795 